\title{
THE DESIGN AND PERFORMANCE OF THE INTEGRATED SPALLATION NEUTRON SOURCE VACUUM CONTROL SYSTEM*
}

\author{
J. Tang ${ }^{\#}$, P. Ladd, D. Williams, J. Crandall, ORNL, Oak Ridge, TN 37831, USA
}

\section{Abstract}

The Spallation Neutron Source (SNS) vacuum control systems have been developed within a collaboration of Lawrence Berkeley National Laboratory (LBNL), Los Alamos National Laboratory (LANL), Thomas Jefferson National Accelerator Facility (TJNAF), and Brookhaven National Laboratory (BNL). Each participating lab is responsible for a different section of the machine: LBNL for the Front-End section, LANL for the warm LINAC section, TJNAF for the cold LINAC section and BNL for the Ring section. Although a great deal of effort has been made to standardize vacuum instrumentation, components and global control system interfaces, the varied requirements of the different sections of the machine have made horizontal integration of the individual vacuum control systems both interesting and challenging. To support machine commissioning, the SNS control and vacuum teams have developed and implemented a series of test strategies and interlock schemes that have allowed horizontal vacuum system integration to be achieved in an effective manner. The design of the vacuum control system interlock schemes developed will be discussed together with the results of performance measurements made and experience gained in developing real-time control with an industrial Ethernet for this application.

\section{INTRODUCTION}

The success of the SNS is measured by the high availability of all systems during operation including vacuum subsystems and their components. Beam energy increases as it progress through from the ion source to final delivery of the beam on the target with vacuum operating levels varying to support physics requirements. (see table 1) [1]. The design of the SNS vacuum system and its control system has been achieved through a multilaboratory collaboration. To maintain the necessary high reliability, availability and flexibility of the vacuum system, both Programmable Logic Controllers (PLCs) and EPICS Input and Output Controllers (IOCs) are used for controlling and monitoring the vacuum devices except for the superconducting vacuum control system that required a fast response time for the sector gate valves (SGVs) [6]. Although a great deal of effort has been taken to standardize vacuum instrumentation, components and global control system interfaces [2], two major challenges

* SNS is managed by UT-Battelle, LLC, under contract DE-AC0500OR22725 for the U.S. Department of Energy. SNS is a collaboration of six US National Laboratories: Argonne National Laboratory (ANL), Brookhaven National Laboratory (BNL), Thomas Jefferson National Accelerator Facility (TJNAF), Los Alamos National Laboratory (LANL), Lawrence Berkeley National Laboratory (LBNL) and Oak Ridge National Laboratory (ORNL).

\#jtang@ornl.gov had to be overcome for the successful integration of the individual vacuum control systems delivered by different partner labs.

These were:

- The use of different vacuum control schemes due to the varied requirements of the different sections of the machine and the different experience of the design engineers.

- The control handling for the "Gray" areas in between the two adjacent subsystems usually where a sector gate valve (SGV) separating them requires to know the vacuum status at ether side.

Table 1: SNS Vacuum Level Requirements

\begin{tabular}{|l|l|l|l|}
\hline Subsystems & $\begin{array}{l}\text { Vacuum } \\
\text { Levels }\end{array}$ & $\begin{array}{l}\text { Beam } \\
\text { Energy }\end{array}$ & $\begin{array}{l}\text { Number of } \\
\text { Vacuum } \\
\text { Devices } \\
\text { Controlled }\end{array}$ \\
\hline IS/LEBT & $1 \mathrm{e}-4$ Torr & $65 \mathrm{KeV}$ & 25 \\
\hline RFQ & $1 \mathrm{e}-6$ Torr & $2.5 \mathrm{MeV}$ & 51 \\
\hline MEBT & $5 \mathrm{e}-7$ Torr & $2.5 \mathrm{MeV}$ & 29 \\
\hline DTL & $1.8 \mathrm{e}-7$ Torr & $86.8 \mathrm{MeV}$ & 107 \\
\hline CCL & $1.4 \mathrm{e}-7$ Torr & $185.6 \mathrm{MeV}$ & 141 \\
\hline SCL & $1 \mathrm{e}-9$ Torr & $1 \mathrm{GeV}$ & 274 \\
\hline HEBT & $5 e-8$ Torr & $1 \mathrm{GeV}$ & 39 \\
\hline Ring & $1 \mathrm{e}-8$ Torr & $1 \mathrm{GeV}$ & 106 \\
\hline RTBT & $1 \mathrm{e}-7$ Torr & $1 \mathrm{GeV}$ & 36 \\
\hline
\end{tabular}

To support machine commissioning and allow horizontal vacuum system integration, a set of test procedures and interlock schemes were developed for the delivered systems. In this paper, we describe the vacuum interlock schemes and their performance to overcome the challenges described above. In addition, we also discuss the experience and lessons learned of in developing an industrial Ethernet with real-time control for this application.

\section{ARCHITECTURE}

With the exception of superconducting vacuum control system, which uses customized control boxes to achieve fast beam line vacuum trip interlock responses needed, the rest of the control system architecture uses a classic three layers of distributed real-time control system: Device Control Layer, equipped with Allen-Bradley ControlLogix Programmable Logic Controllers (PLC) [7] to monitor gauge and pump set 


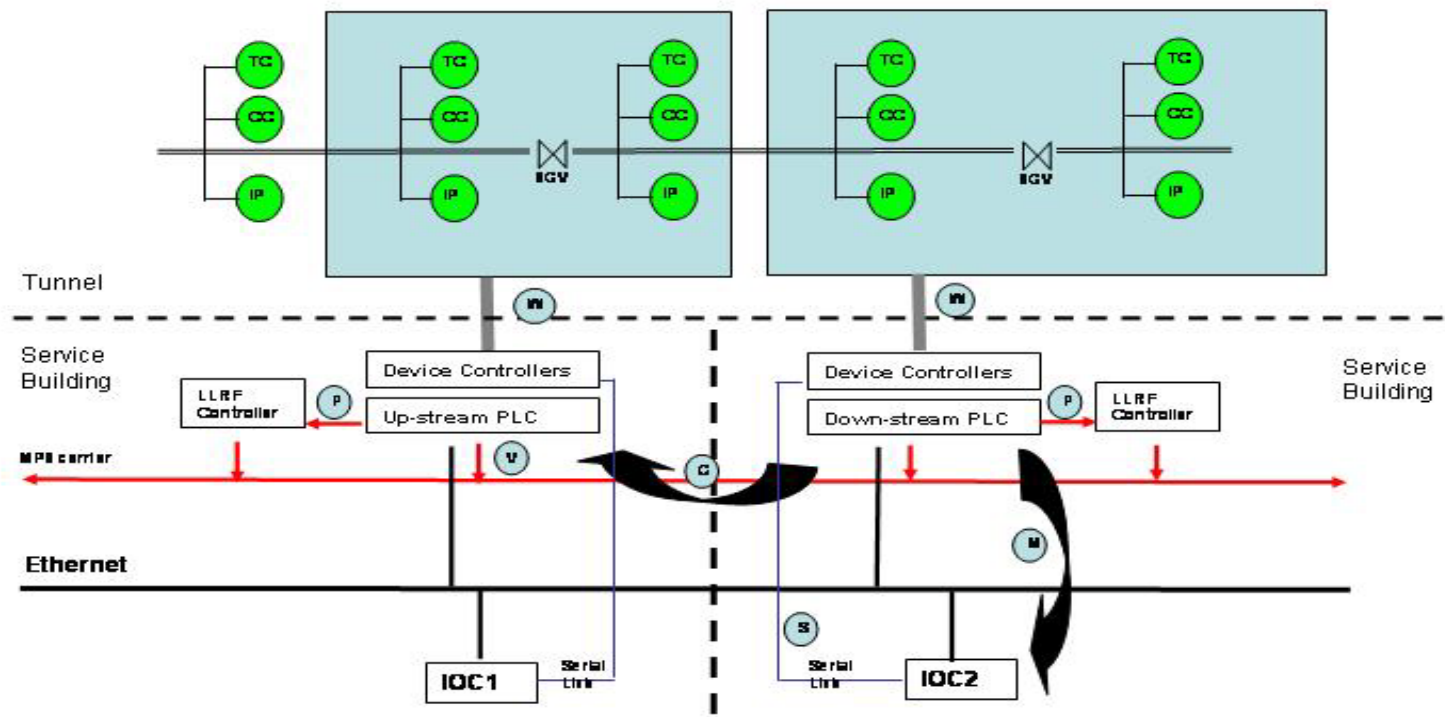

Figure 1: SNS Vacuum Interlock Scheme where "w" is noted as hard wired devices to local PLC.

point outputs and control valves; Global Control Layer, equipped with an EPICS Input Output Controller (IOC) to overlook multiple subsystems; and Operator Interface Layer, equipped with Linux workstations to provide machine operation interface.

\section{INTERLOCK SCHEME}

The major contribution from the SNS vacuum and control teams has been the development and integration of an interlock scheme between two subsystems divided by a Sector Gate Valve (SGV) that is highly reliable, protects the vacuum integrity of each subsystems and equipments as well as providing the flexibility necessary to maximize the availability. Figure 1 shows a typical interlock scheme employed in the SNS vacuum subsystems.

\section{Interlock Scheme Design}

Two methods are used in this interlock scheme: (i) use directly hard wire vacuum sensors on the upstream and downstream of a SGV to one PLC. This is to avoid dependency of inter-process communication for the critical data passing and provide high reliability, noted as "w" in Figure 1; (ii) use EtherNet/IP [3][7] producerconsumer protocol link to provide a redundant interlock vote scheme for higher machine availability. This is to prevent machine down time caused by a single vacuum sensor device failure and to reduce costs on hard wiring multiple devices. It provides an option to temporarily bypass a knowing bad device sensor in an interlock chain. A significant studies and field test measurements have been made on the interlock link based on real-time IO via Ethernet and its deterministic performance.

\section{Real-time Interlock via Ethernet}

Real-time is an assessment of a system's ability to respond to external events. "Hard" or "soft" real-time is based the latency tolerance of a specific application. In SNS vacuum control system, Allen-Bradley Controllogix PLCs are used for local device control and interlock process. A producer-consumer model is utilized to performance inter-PLC communication via Ethernet link. A fail-safe or timeout scheme is implemented within the PLCs for exceptional Network latency handling.

\section{Multicast and IGMP Snooping}

The multicast is used in Allen-Bradley ControlLogix producer-consumer model which enables multiple consumers to receive the same information from a producer of data. This reduces the usage of network bandwidth and minimizes latency. The IGMP snooping is a method that the modern network switch uses to send data only to the ports where the consumers are located. The SNS vacuum control system takes advantages of these modern techniques. It saves the project cost on hardwiring of many interlock devices and in the meantime, increases configuration flexibility and machine availability.

\section{FIELD TEST MEASUREMENT RESULTS}

A series of test measurements on the interlock response time has been conducted to verify the interlock scheme performance. Figure 2 shows the test results for the SNS DTL vacuum to beam interlock response performance. 
DTL Vacuum MPS Interlock Response Time

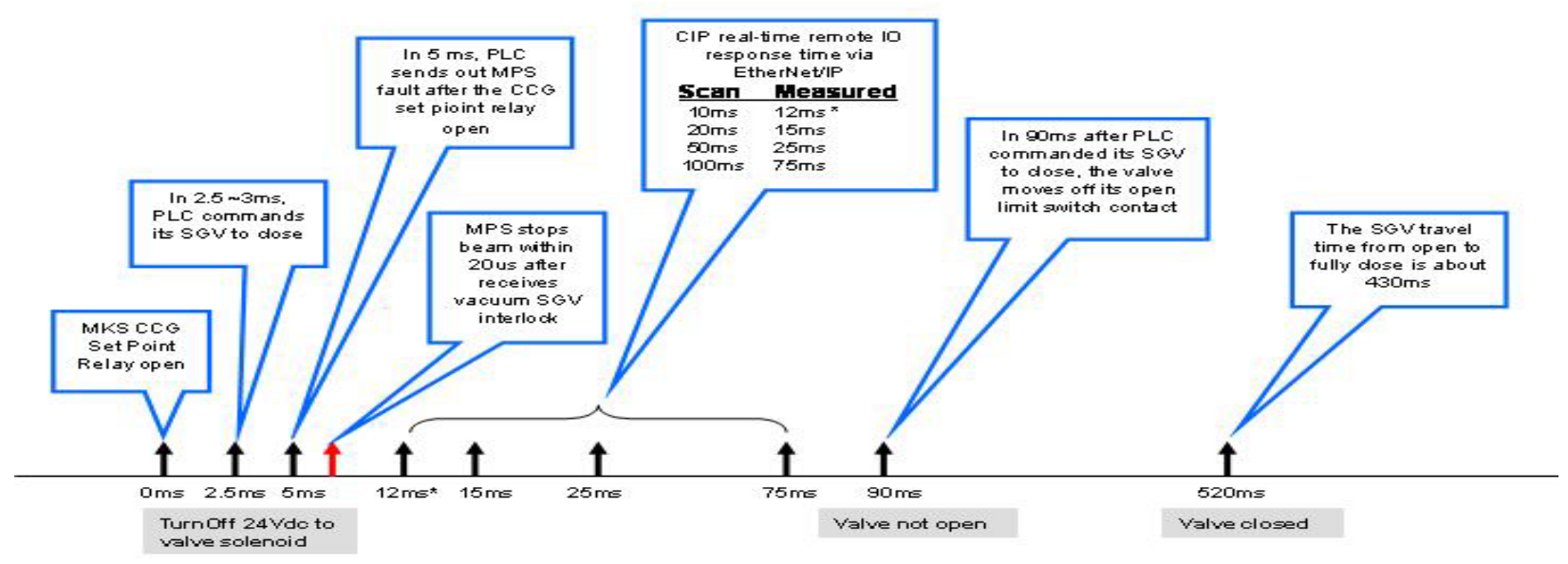

Figure 2: SNS DTL and CCL vacuum MPS interlock response time.

Based on the test measurement results, we readjusted the PLC scan rate to optimize and balance between the process CPU usage and interlock response time.

\section{SUMMARY}

The SNS vacuum control system is a cooperative endeavor among six DOE Labs. Even though a great deal of effort has been made on standardization among SNS vacuum subsystems, the varied requirements of the different sections of the machine made horizontal integration of the individual vacuum control systems challenging, especially in the "gray" area where the subsystems are divided. To support machine integration, an interlock scheme has been developed to mitigate the control logic "gaps" between the subsystems from different collaborating labs. The scheme utilizes modern network technology aimed at archiving high reliability and availability.

Our commissioning experience suggests that careful control of system network configuration changes through a reliable configuration management plan will make significant contribution to high machine reliability and availability.

We successfully delivered beam to the end of warm linac last year. The commissioning continues with beam scheduled to be delivered to the end of superconducting linac by August this year and to target in 2006.

\section{ACKNOWLEDGEMENT}

The authors would like to acknowledge the valuable contributions from many members of LANL control team,
JLAB instrumentation group, and the SNS Accelerator Division vacuum and control system teams. Among them, Pilar Maroquin, Charles Pinney and Bobby Quintana, who supported us on-site during machine initial checkout, and Randal McPherson and Bill Devan, who helped with the control system function checkout and provided many valuable suggestions on the control logic.

\section{REFERENCES}

[1] P. Ladd, et al, "An Overview of the Spallation Neutron Source Vacuum Systems," Proceedings of AVS 04.

[2] J. Tang, et al, "SNS Vacuum Instrumentation and Control System," ICALEPCS'01, San Jose, November, 2001.

[3] K. Kasemir, et al, "Interfacing the ControlLogix PLC over EtherNet/IP," ICALEPCS'01, San Jose, November, 2001.

[4] J. Bernardin, et al, "SNS Drift Tube Linac Vacuum System Final Design Report," SNS-104020400DE0001, LLNL and LANL, January, 2001.

[5] J. Bernardin, et al, "SNS Coupled Cavity Linac Vacuum System Final Design Report," SNS104020400-DE0001, LLNL and LANL, January, 2001.

[6] W. Schneider, et al, "Design of the SNS Cryomodule," PAC'01, Chicago, June, 2001.

[7] http://www.ab.com

[8] http://www.cisco.com 